\title{
KHẢO SÁT QUY TRÌNH THÀNH LÂP LƯỚI GPS/GNSS TRONG XÂY DỰNG CÔNG TRÌNH ĐƯỜNG HẦM
}

\author{
TS. PHẠM QUÓC KHÁNH(1), ThS. VÕ NGỌC DŨNG ${ }^{(1)}$, \\ KS. NGUYẼ̃N VĂN THÀNH(2) \\ (1)Trường đại học Mỏ - Địa chất \\ (2)Trường đại học Tài nguyên và Môi trường
}

\section{1. Đặt vấn đề}

Trong quá trình thiết kế, thi công và khai thác công trình ngầm nói chung và công trình đường hầm nói riêng, công tác trắc địa đóng vai trò quan trọng. Độ chính xác của các phép đo đạc và tính toán là nhân tố quyết định hiệu quả kinh tế-kỹ thuật của quá trình xây dựng đường hầm. Kết quả thông hướng đường hầm được thể hiện qua hai thành phần: độ chính xác hướng ngang và độ chính xác hướng dọc đường hầm, trong đó thông hướng ngang là đối tượng cần được được quan tâm hơn với yêu cầu độ chính xác cao hơn [1]. Sự sai lệch về hướng ngang vượt quá giới hạn cho phép so với thiết kế sẽ gây đình trệ sản xuất, tổn thất về kinh phí, thậm chí gây nguy hiểm tính mạng con người. Các công tác đo đạc phục vụ thi công trong đường hầm đều phải dựa vào mạng lưới khống chế trắc địa trên mặt đất. Chất lượng của mạng lưới này là yếu tố quết định độ chính xác và độ tin cậy của các phép cho hướng và kết quả thông hướng đường hầm. Trước đây, lưới khống chế mặt bằng trên mặt đất thường được thành lập bằng các phương pháp truyền thống dưới dạng lưới đo góc-cạnh như: các phương pháp giao hội, chuỗi tam giác, lưới đường chuyền .v.v... Các phương pháp này đòi hỏi nhiều thời gian và công sức, chất lượng mạng lưới bị suy giảm trong trường hợp đường hầm được thi công trong các điều kiện đia hình phức tạp, sông suối chia cắt .v.v...Trong thời gian gần đây, với sự phát

Ngày nhận bài: 19/10/2015 triển của hệ thống GNSS, các mạng lưới khống chế trắc địa nói chung và lưới khống chế phục vụ xây dựng đường hầm nói riêng đã được thành lập bằng công nghệ hiện đại này. Tính ưu việt của phương pháp đã được minh chứng trong thực tiễn, tuy vậy, chưa có quy chuẩn cụ thể nào quy định quy trình thiết kế, và thi công lưới khống chế mặt bằng trong xây dựng công trình đường hầm bằng công nghệ GPS/GNSS.

\section{Thiết kế lưới GPS trong xây dựng} công trình đường hầm

\subsection{Nguyên tắc chọn điểm lưới khống chế mặt bằng}

Xuất phát từ đặc điểm của công tác trắc địa phục vụ thi công đường hầm, vị trí điểm lưới khống chế có những yêu cầu riêng. Để bảo đảm độ tin cậy công tác đo chuyền tọa độ cho mạng lưới khống chế trong đường hầm, tại vị trí cửa hầm và các lối đào phụ (giếng đứng, giếng nghiêng, hầm phụ bằng, hầm phụ nghiêng...) cần phải có ít nhất 3 điểm khống chế mặt bằng. Điểm ở cửa hầm phải thuận lợi cho việc bố trí trục thi công, đo nối điểm khống chế trên mặt đất với điểm khống chế trắc địa trong hầm; chiều dài canh định hướng để định hướng đường hầm không nên nhỏ hơn $300 \mathrm{~m}[1]$.

\subsection{Lựa chọn hệ quy chiếu}

- Chọn hệ trục tọa độ: Đối với lưới khống chế đường hầm, có hai phương án về hệ trục tọa độ: Hệ trục tọa độ quốc gia và hệ

Ngày chấp nhận đăng: 04/11/2015 
tọa độ độc lập. Hệ tọa độ đôc lập là hệ tọa độ quy ước có điểm gốc tọa độ là một điểm ở cửa hầm; trục OY được chọn theo hướng vuông góc với mặt đào thông hầm[1].

- Chọn mặt chiếu: Với các đường hầm sâu hoặc được xây dựng ở vùng núi cao cần phải lựa chọn mặt chiếu lưới thi công cho phù hợp để tính các số hiệu chỉnh khi chuyển các trị đo từ mặt đất tự nhiên xuống ellipxoid quy chiếu và chuyển từ ellipxoid quy chiếu lên mặt phẳng qua phép chiếu Gauss hoặc phép chiếu UTM [2]. Với công trình đường hầm, thường chọn mặt chiếu có độ cao là độ cao trục tim hầm.

\section{3. Ước tính độ chính xác cần thiết của lưới}

Trong các nguồn sai số đào thông hầm, sai số hướng ngang được coi là quan trọng nhất. Khi thiết kế lưới khống chế mặt bằng, cần xuất phát từ yêu cầu sai số thông hầm để đánh giá, ước tính độ chính xác, qua đó xác định các chỉ tiêu kỹ thuật của lưới khống chế trắc địa trên mặt đất. Công thức tổng quát tính sai số trung phương (SSTP) tổng hợp hướng ngang tại vị trí đào thông hầm đối hướng với đường hầm thẳng được thể hiện bằng công thức:

$$
M_{q}=\sqrt{m_{1}^{2}+m_{2}^{2}+m_{3}^{2}+m_{4}^{2}+m_{5}^{2}}
$$

trong đó:

$\mathrm{m}_{1}$ - SSTP hướng ngang của khống chế trắc địa trên mặt đất;

$\mathrm{m}_{2}, \mathrm{~m}_{3}$ - SSTP hướng ngang định hướng đường hầm qua lối đào phụ (nếu có);

$\mathrm{m}_{4}, \mathrm{~m}_{5}$ - SSTP hướng ngang của hai tuyến đường chuyền nhánh trong hầm.

Đối với đường hầm cong, ảnh hưởng của sai số hướng dọc và hướng ngang đối với độ chính xác thông hầm có giá trị như nhau, công thức (1) sẽ có dạng:

$$
\frac{M_{q}}{\sqrt{2}}=\sqrt{m_{1}^{2}+m_{2}^{2}+m_{3}^{2}+m_{4}^{2}+m_{5}^{2}}
$$

Để xác định các nguồn sai số trong (1) và (2), tùy thuộc vào hình dạng đường hầm, có thể sử dụng nguyên tắc ảnh hưởng bằng nhau hoặc không bằng nhau để xác định các nguồn sai số.

\subsection{Tính yếu tố đặc trưng và ước tính độ chính xác lưới}

a. Tính các yếu tố đặc trưng của lưới

- Dựa vào bản đồ địa hình tỷ lệ trung bình (với đường hầm dài) hoặc bản đồ tỷ lệ lớn (với đường hầm ngắn) để thiết kế đồ hình và đồ giải tọa độ gần đúng lưới thiết kế. Các điểm trong lưới phải được chọn theo nguyên tắc nêu trong mục 2.1.

- Thiết kế gốc lưới GPS

- Dựa vào khả năng máy móc và thiết bị (số lượng máy thu) tiến hành tính toán các điều kiện đặc trưng của lưới[3].

Số thời đoạn đo:

$$
C=\frac{n \cdot m}{N}
$$

trong đó:

$\mathrm{n}$ - số điểm trong lưới;

m-số lần đặt máy (đặt trạm) ở mỗi điểm;

$\mathrm{N}$ - số máy thu.

Tổng số đường đáy:

$$
J_{t}=\frac{C \cdot N \cdot(N-1)}{2}
$$

Số đường đáy cần thiết: $J_{c t}=n-1$

Số đường đáy độc lập: $J_{d l}=C(N-1)$

Số đường đáy dư:

$$
J_{d}=C(N-1)-(n-1)
$$

Số cạnh GPS/GNSS trong một thời đoạn đo của đồ hình đồng bộ được tạo thành từ $\mathrm{N}$ máy thu sẽ là: 


$$
J=\frac{N \cdot(N-1)}{2}
$$

Đồ hình lưới GPS/GNSS linh hoạt hơn đồ hình của lưới trắc địa truyền thống do các điểm lưới không cần nhìn thông nhau. Căn cứ vào mục đích sử dụng, thông thường có 4 phương thức cơ bản thành lập lưới là liên kết điểm; liên kết cạnh; liên kết hỗn hợp cạnh-điểm và liên kết lưới.

\section{b. Ước tính độ chính xác lưới}

Theo nguyên lý đo GPS/GNSS tương đối, gia số tọa độ giữa các điểm đặt máy thu sẽ được xác định, từ đó có thể tính được cạnh và phương vị giữa các điểm đo. Nếu coi đây là các trị đo độc lập, sẽ ước tính được độ chính xác lưới GPS/GNSS dựa trên thuật toán bình sai gián tiếp quen thuộc. Có hai phương pháp ước tính ảnh hưởng của lưới trên mặt đất đối với độ chính xác đào thông hầm, bao gồm:

- Ước tính theo sai số hướng ngang điểm cuối chuỗi, tức là lấy SSTP hướng ngang điểm cửa hầm còn lại làm giá trị ảnh hưởng sai số lưới khống chế trên mặt đất đối với độ chính xác hướng ngang đào thông hầm.

- Ước tính theo sai số hướng ngang điểm đào thông hầm, còn gọi là phương pháp "e-lip sai số điểm không", lấy ảnh hưởng sai số lưới khống chế trên mặt đất đối với độ chính xác hướng ngang đào thông hầm đối hướng là hình chiếu của e-lip sai số điểm "không" trên mặt đào thông hầm được tính theo công thức:

$$
M_{q}^{2}=E^{2} \cos ^{2} \Psi+F^{2} \sin ^{2} \Psi
$$

trong đó:

$\Psi$ là góc phương vị của trục $X$ khi lấy bán trục lớn của ellipse làm hướng khởi đầu,

$$
\Psi=360^{\circ}-\varphi_{0} \text {; }
$$

$E, F, \varphi_{0}$ là các yếu tố của ellipse sai số tương hỗ vị trí điểm "không".
Sau khi ước tính độ chính xác lưới thiết kế cần so sánh với độ chính xác cần thiết để có thể kết luận xem lưới thiết kế có đủ độ chính xác không hay cần điều chỉnh.

\section{Thi công và xử lý số liệu}

Sau khi ước tính độ chính xác lưới, tiến hành triển khai thi công lưới GPS ngoài thực địa với các nội dung chính sau đây:

- chọn điểm, chôn mốc theo thiết kế;

- lập lịch đo;

- đo đạc;

- xử lý số liệu.

Quy trình thành lập lưới khống chế mặt bằng thi công đường hầm bằng công nghệ GPS/GNSS trên đây được minh họa theo sơ đồ như sau: (Xem hình 1)

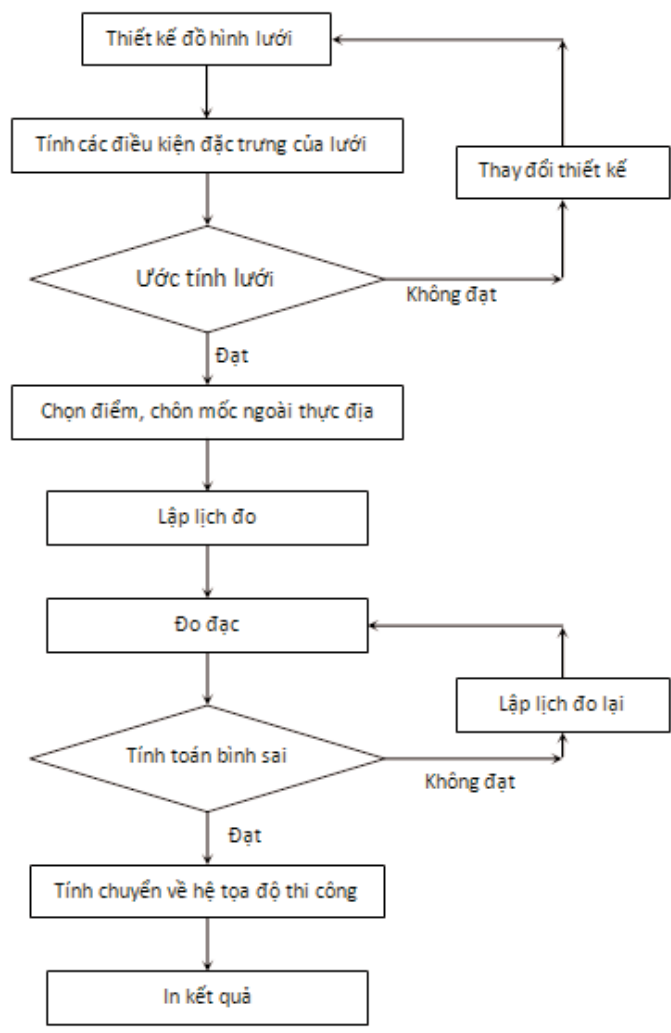

Hình 1: So đồ quy trình thành lập lưới GPS phục vụ thi công đường hầm 


\section{Thực nghiệm}

Mô hình thực nghiệm là một đường hầm vừa thẳng vừa cong có hai cửa hầm là $\mathrm{A}_{1}$ và $C_{1}$ được chia làm 2 đoạn bởi giếng đứng $\mathrm{G}_{1}$, đoạn hầm thẳng dài khoảng $2.2 \mathrm{~km}$ và đoạn hầm cong dài khoảnh $1.3 \mathrm{~km}$ như hình 2. (Xem hinh 2)

\section{1. Ước tính độ chính xác cần thiết của lưới}

Chọn hệ trục tọa độ có điểm gốc $O$ trùng điểm $A_{1}$, trục $Y$ trùng đường nối giữa hai cửa hầm, khi đó phải dựa vào công thức đường hầm cong để tính cho cả 2 đoạn hầm.

Ký hiệu các nguồn sai số ảnh hưởng tới độ chính xác hướng ngang đào thông hầm đối hướng đoạn hầm $A_{1} G_{1}$ là $m_{11}, m_{12}$, $m_{14}, m_{15}$; của đoạn hầm $\mathrm{G}_{1} \mathrm{C}_{1}$ là $m_{21}, m_{22}$, $m_{24}, m_{25}$. Khi đó sai số hướng ngang cần thiết của khống chế trên mặt đất là $M_{q m d}$ :

$$
M_{q m d}^{2}=m_{11}^{2}+m_{21}^{2}
$$

Với $m_{11}$ và $m_{12}$ được tính từ công thức tính SSTP tổng hợp hướng ngang tại chỗ đào thông hầm đối hướng của đường hầm cong:

$$
\frac{M_{q 1}^{2}}{2}=m_{11}^{2}+m_{12}^{2}+m_{14}^{2}+m_{15}^{2}
$$

Áp dụng nguyên tắc ảnh hưởng bằng nhau đối với các nguồn sai số thành phần, đoạn hầm $A_{1} G_{1}$ dài $1,3 \mathrm{~km}$ nên $M_{q 1}=50 \mathrm{~mm}$ [1], suy ra:

$$
m_{11}=\frac{M_{q 1}}{\sqrt{2.4}}=\frac{50}{\sqrt{8}}=17,7 \mathrm{~mm}
$$

Tương tự với đoạn hầm $\mathrm{G}_{1} \mathrm{C}_{1}$ ta có: $m_{21}$ $=17,7 \mathrm{~mm}$

Thay vào công thức (10) có: $\mathrm{M}_{\mathrm{qmd}}=$ $25 \mathrm{~mm}$

Như vậy cần thành lập lưới khống chế mặt bằng trên mặt đất với sai số hướng ngang không quá $25 \mathrm{~mm}$.

\subsection{Thiết kế đồ hình lưới}

Lưới khống chế mặt bằng trên mặt đất dự kiến được thành lập bằng công nghệ GPS với 5 máy thu của hãng Trimble-Mỹ. Theo phần 2.1, tại cửa hầm và lối đào phụ bắt buộc phải có 3 điểm khống chế mặt bằng; cạnh nối giữa hai cửa hầm và lối đào phụ phải được xác định với độ chính xác cao nhất, (tức được đo nhiều lần nhất). Từ các nguyên tắc trên, lưới khống chế trên mặt đất cần được thiết kế với các điểm ở hai cửa hầm và miệng giếng đứng là $\mathrm{A}_{1}, \mathrm{C}_{1}$ và $\mathrm{G}_{1}$, các điểm định hướng tại hai cửa hầm và giếng đứng bố trí như hình 2 . Trong đó điểm $A_{2}$ và $A_{3}$ là 2 điểm định hướng cho điểm $A_{1}$, điểm $G_{2}$ và $G_{3}$ là 2 điểm định hướng cho điểm $G_{1}$, điểm $C_{2}$ và $C_{3}$ là 2 điểm định hướng cho điểm $\mathrm{C}_{1}$. Điểm định hướng phải nhìn thấy điểm ở cửa hầm, điểm ở miệng giếng đứng và không nhất thiết phải thông hướng với nhau. Các điểm định hướng nên cách xa điểm cửa hầm và

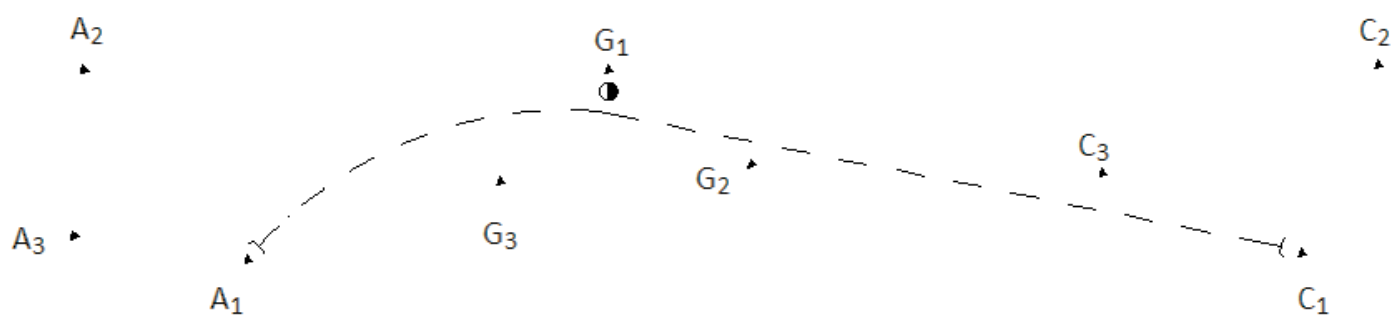

Hình 2: Mô hình đường hầm 
điểm ở miệng giếng ít nhất là 300m.

\subsection{Tính điều kiện đặc trưng lưới GPS}

Áp dụng các công thức từ 3 đến 8 , ta có:

Số thời đoạn đo: $C=\frac{n \cdot m}{N}=\frac{9 \cdot \frac{15}{9}}{5}=3$

Tổng số đường đáy :

$$
J_{t}=\frac{C \cdot N \cdot(N-1)}{2}=\frac{3 \cdot 5 \cdot(5-1)}{2}=30
$$

Số đường đáy cần thiết: $J_{C t}=n-1=9-1=$ 8

Số đường đáy độc lập: $J_{d l}=C(N-1)=$ $3(5-1)=12$

Số cạnh du: $J_{d}=C(N-1)-(n-1)=3(5-$ 1) $-(9-1)=4$

Số cạnh GPS trong một thời đoạn đo của đồ hình đồng bộ được tạo thành từ $\mathrm{N}$ máy thu sẽ là:

$$
J=\frac{N \cdot(N-1)}{2}=\frac{5(5-1)}{2}=10
$$

Số vòng đo đồng bộ ít nhất là:

$$
T=J-(N-1)=\frac{(N-1)(N-2)}{2}=10-(5-1)=6
$$

Như vậy, lưới được thiết kế 3 ca đo và có sơ đồ như hình 3. (Xem hình 3)

\section{4. Ước tính độ chính xác lưới}

Theo [1], ước tính ảnh hưởng sai số lưới trên mặt đất đối với độ chính xác hướng ngang đào thông hầm đối hướng the phương pháp "elippse sai số điểm không" có độ chính xác tốt hơn. Kết quả ước tính theo phương pháp này đối với lưới GPS thiết kế trình bày trong bảng 1 .

(Xem bảng 1, 2)
4.5. Đo đạc và xử lý số liệu thực nghiệm

Lưới thực nghiệm được đo trên mô hình tại khu vực đô thị mới Mỹ Đình bằng 5 máy thu GPS (3 máy Trimble R3 và 2 máy Trimble 4600LS). Tiến hành đo $3 \mathrm{ca}$, mỗi ca 60 phút, các thông số khi đo so với điều kiện lập lịch đều tốt (số vệ tinh luôn > 6, PDOP < 5)[3].

Sử dụng phần mềm xử lý số liệu GPS TBC (Trimble Business Center) để xử lý số liệu đo thực tế, các cạnh trong lưới đều có lời giải tốt nên tiến hành bình sai lưới. Trong lưới có điểm $\mathrm{G}_{1}$ có tọa độ nhà nước nên lấy làm điểm gốc, kết quả bình sai trình bày trong bảng 3 .

Nhận xét: Kết quả bình sai lưới GPS và kết quả ước tính lưới tương đối sát nhau, sai lệch không đáng kể cho thấy tác dụng của việc ước tính lưới trước khi đo.

Lưới mặt bằng xây dựng đường hầm thành lập bằng GPS cho độ chính xác cao, sai số vị trí điểm nhỏ hơn rất nhiều so với sai số cho phép.

Vì đây là mô hình đường hầm nên không tính chuyển về hệ tọa độ công trình.

\section{Kết luận}

- Thành lập lưới mặt bằng trên mặt đất trong thi công đường hầm bằng công nghệ GPS nên tuân thủ theo đúng quy trình để lưới đảm bảo độ chính xác theo yêu cầu.

- Cần ước tính lưới GPS trước khio tiến hành đo đạc lưới.

- Lưới mặt bằng trên mặt đất trong xây dựng đường hầm không những đảm bảo độ chính xác mà còn có lượi về thời gian và chi phí. $O$ 


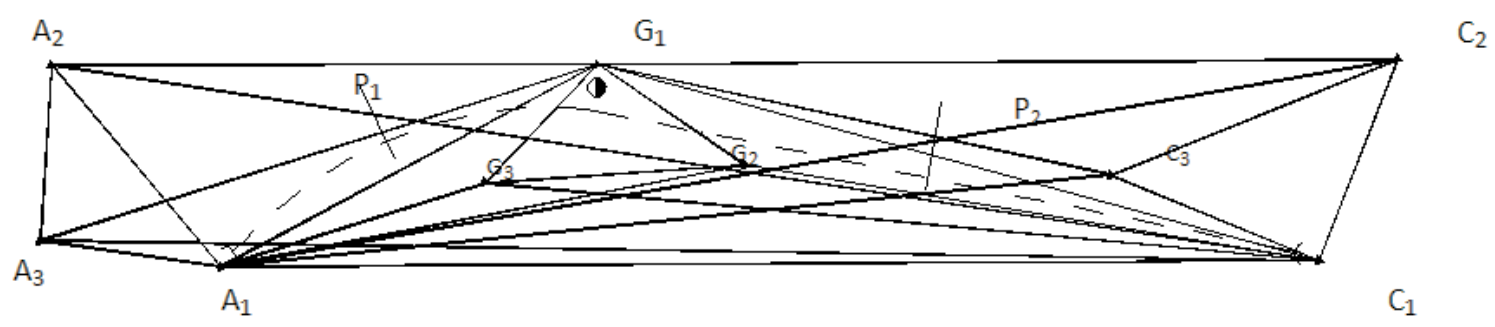

Hình 3: Sơ đồ lưới GPS

Bảng 1: Sai số vị trí điểm lưới GPS

\begin{tabular}{|c|c|c|c|c|c|c|l|}
\hline STT & Tên điểm & $\mathbf{X}(\mathbf{m})$ & $\mathbf{Y}(\mathbf{m})$ & $\mathbf{m}_{\mathbf{x}}(\mathbf{m m})$ & $\mathbf{m}_{\mathbf{y}}(\mathbf{m m})$ & $\mathbf{m}_{\mathbf{p}}(\mathbf{m m})$ & Ghi chú \\
\hline 1 & $\mathrm{~A}_{1}$ & 2324128,5 & 502148,0 & & & & Cửa hầm \\
\hline 2 & $\mathrm{~A}_{2}$ & 2323789,5 & 501716,5 & 2,3 & 2,9 & 3,7 & \\
\hline 3 & $\mathrm{~A}_{3}$ & 2323748,0 & 501990,0 & 2,4 & 2,1 & 3,2 & \\
\hline 4 & $\mathrm{G}_{1}$ & 2325304,0 & 501414,0 & 2,0 & 2,4 & 3,1 & Giếng đứng \\
\hline 5 & $\mathrm{G}_{2}$ & 2325712,5 & 501444,0 & 3,0 & 2,8 & 4,1 & \\
\hline 6 & $\mathrm{G}_{3}$ & 2324794,0 & 501715,5 & 2,8 & 2,8 & 4,0 & \\
\hline 7 & $\mathrm{C}_{1}$ & 2327420,5 & 501977,0 & 2,0 & 3,7 & 4,2 & Cửa hầm \\
\hline 8 & $\mathrm{C}_{2}$ & 2327640,5 & 501535,0 & 2,7 & 4,3 & 5,1 & \\
\hline 9 & $\mathrm{C}_{3}$ & 2327078,5 & 501789,5 & 2,8 & 3,9 & 4,8 & \\
\hline 10 & $\mathrm{P}_{1} \mathrm{~A}$ & 2324716,5 & 501781,0 & 1,6 & 2,6 & 3,1 & Điểm đào thông \\
\hline 11 & $\mathrm{P}_{1} \mathrm{G}$ & 2324716,5 & 501781,0 & 2,5 & 3,7 & 4,5 & Điểm đào thông \\
\hline 12 & $\mathrm{P}_{2} \mathrm{G}$ & 2326362,5 & 501695,5 & 2,3 & 4,9 & 5,4 & Điểm đào thông \\
\hline 13 & $\mathrm{P}_{2} \mathrm{C}$ & 2326362,5 & 501695,5 & 2,3 & 5,6 & 6,1 & Điểm đào thông \\
\hline
\end{tabular}

Bảng 2: Elipse sai số tương hỗ vị trí điểm đào thông hầm

\begin{tabular}{|c|c|c|c|c|c|}
\hline Điểm 1 & Điểm 2 & $\mathbf{E}(\mathbf{m m})$ & $\mathbf{F}(\mathbf{m m})$ & $\varphi_{0}\left(0^{\prime \prime}\right)$ & $\mathbf{m q}(\mathbf{m m})$ \\
\hline $\mathrm{P}_{1} \mathrm{~A}$ & $\mathrm{P}_{1} \mathrm{G}$ & 4,9 & 7,2 & 3420320,9 & 4,7 \\
\hline $\mathrm{P}_{2} \mathrm{G}$ & $\mathrm{P}_{2} \mathrm{C}$ & 6,9 & 3,0 & 3545324,2 & 6,9 \\
\hline
\end{tabular}

Kết quả ước tính cho thấy, lưới GPS thiết kế đạt độ chính xác theo yêu cầu. 
Bảng 3: Kết quả bình sai lưới GPS

\begin{tabular}{|c|c|c|c|c|c|c|c|}
\hline \multirow{2}{*}{} & \multirow{2}{*}{ Tên điểm } & \multicolumn{2}{|c|}{ Tọa độ bình sai } & \multicolumn{3}{c|}{ Sai số } & Ghi chú \\
\cline { 3 - 8 } & & $\mathbf{X}(\mathbf{m})$ & $\mathbf{Y}(\mathbf{m})$ & $\mathbf{m}_{\mathbf{x}}(\mathbf{m m})$ & $\mathbf{m}_{\mathbf{y}}(\mathbf{m m})$ & $\mathbf{m}_{\mathbf{p}}(\mathbf{m m})$ & \\
\hline 1 & $\mathrm{~A}_{1}$ & 2324128,3177 & 502148,1481 & 1,0 & 1,0 & 1,4 & \\
\hline 2 & $\mathrm{~A}_{2}$ & 2323789,4756 & 501716,4060 & 2,4 & 2,5 & 3,5 & \\
\hline 3 & $\mathrm{~A}_{3}$ & 2323748,0282 & 501989,8016 & 1,8 & 1,9 & 2,6 & \\
\hline 4 & $\mathrm{G}_{1}$ & 2325304,0000 & 501414,0000 & & & & Fixed \\
\hline 5 & $\mathrm{G}_{2}$ & 2325712,3322 & 501444,5244 & 1,7 & 2,5 & 3,0 & \\
\hline 6 & $\mathrm{G}_{3}$ & 2324794,0213 & 501715,5970 & 2,8 & 3,71 & 4,6 & \\
\hline 7 & $\mathrm{C}_{1}$ & 2327420,2467 & 501976,9316 & 1,1 & 1,1 & 1,6 & \\
\hline 8 & $\mathrm{C}_{2}$ & 2327640,5342 & 501534,9096 & 1,8 & 1,9 & 2,6 & \\
\hline 9 & $\mathrm{C}_{3}$ & 2327078,5697 & 501789,4522 & 1,9 & 1,9 & 2,7 & \\
\hline
\end{tabular}

\section{Tài liệu tham khảo}

[1]. Phan Văn Hiến, 2014. Trắc địa công trình đường hầm, NXB Xây dựng.

[2]. Phạm Quốc Khánh, 2007. Nghiên cứu ứng dụng công nghệ mới trong trắc địa công trình đường hầm, Luận văn thạc sĩ kỹ thuật, Đại học Mỏ-Địa chất.

[3]. Trần Viết Tuấn và nnk, 2007. Nghiên cứu ứng dụng GPS trong trắc địa công trình, Đề tài NCKH cấp Bộ, mã số B2005-36-75. 0

\section{Summary}

\section{On the GPS/GNSS network establishment procedure for undergrounds construc- tion}

Dr. Pham Quoc Khanh, MSc. Vo Ngoc Dung, Hanoi University of Mining and Geology

Eng. Nguyen Van Thanh, Hanoi University of Natural Resources and Environment

In underground construction, the lateral error of cut-through play an important role. The value of this error is depended on the quality of control network on the earth surface. The GPS/GNSS control network is more and more applied for underground construction, however, until now there is no standardized procedure on its establishment. The research results on the GPS/GNSS control network establishment procedure have been presented in this paper. $O$ 